\title{
Dificuldades e estratégias de estudantes de pós-graduação da área de ciências da saúde para escrever e publicar em inglês - um estudo qualitativo descritivo.
}

Difficulties and strategies of graduate students in the field of health sciences to write and publish in English - a descriptive qualitative study.

Dificultades y estrategias de losestudiantes de postgradoenel área de ciencias de lasalud a escribir y publicar enInglés - un estudio cualitativo descriptivo.

\author{
Alexandre CUNHA ${ }^{1}$ \\ Alessandro de Oliveira SANTOS ${ }^{2}$ \\ Álvaro Machado DIAS ${ }^{3}$ \\ Beny LAFER ${ }^{4}$ \\ Geraldo F. BUSATTO ${ }^{1,2}$
}

RESUMO: Estudo qualitativo descritivo que descreve dificuldades e estratégias de estudantes de um programa de pós-graduação em medicina para escrever e publicar artigos, derivados de seus trabalhos de mestrado e doutorado, em periódicos indexados pelo JCR-ISI Web ofKnowledge; com base na análise de conteúdo de respostas a um questionário aplicado com 43 estudantes. As dificuldades destacadas foram a falta de treino na escrita em língua inglesa, a compreensão

1 Departamentode Psiquiatria, Universidadede São Paulo,Faculdade de Medicina, São Paulo, Brasil - Núcleo de Apoio à Pesquisa em Neurociência Aplicada (NAPNA), Universidadede São Paulo, São Paulo, Brasil.Contato: alecunhaace@ig.com.br

2 Instituto de Psicologia, Universidadede São Paulo, São Paulo, Brasil

3 Laboratório de Neurociência Clínica, Universidade Federal de São Paulo (UNIFESP, Universidade Federal de São

Paulo), São Paulo, Brasil - Núcleo de Apoio à Pesquisa em Neurociência Aplicada (NAPNA), Universidadede São

Paulo, São Paulo, Brasil

4 Instituto de Psicologia, Universidadede São Paulo, São Paulo, Brasil Contato: geraldo.busatto@gmail.com

ISSN 1982-8829

Tempus, actas de saúde colet, Brasília, 8(3), 145-155, set, 2014// 
e cumprimento de normas dos periódicos e a tradução de termos científicos. As estratégias utilizadas são a leitura de artigos em inglês, a redação do artigo desde o início em língua inglesa e a organização de uma estrutura básica do texto. Além disso, os estudantes tem recebido ajuda de revisores, nacionais e estrangeiros, como orientadores, coorientadores e revisores profissionais em língua inglesa, configurando uma rede de apoio de suporte a essa atividade. Palavras chave: Ciência. Pesquisa. Publicação Científica. Língua Inglesa. Análise de Conteúdo.

ABSTRACT: Descriptive and qualitative study describing difficulties and strategies of students in a program of graduate in psychiatry to write and publish articles derived from their dissertations/ thesis in journals indexed by ISI Web of Knowledge and based on content analysis of responses to a questionnaire answered by 43 students. Difficulties highlighted were the lack of training in writing in English language, understanding and compliance of the journals requirements and the translation of scientific terms. The strategies used are: reading articles in English, the wording of article from the beginning in English and the organization of a basic structure of the text. In addition, students have received help from reviewers, domestic and foreign, as mentors, co-mentors and professionals in English translation/editing, setting up a supporting network to this activity. Key words: Science. Research. Scientific Publication. English Language. Content Analysis.

RESUMEN: Estudio descriptivo, cualitativo describelas dificultades y las estrategias de losestudiantesenun programa de laredacción de artículos médicos y publicación de posgrado derivados de su maestro de obra y doctoradoen revistas indexadas por el JCR de ISI Web ofKnowledge; basadoenelanálisis de contenido de las respuestas a uncuestionario aplicado a 43 estudiantes. Dificultadesdestacaronfueronla falta de formaciónenla escritura enel idioma Inglés, lacomprensión y el cumplimiento de las revistas y latraducción de los términos científicos. Las estrategias utilizadas estánleyendo artículos enInglés, laredaccióndel artículo desde el principio enInglésy laorganización deunaestructura básica del texto.Además, losestudianteshanrecibidoayuda de los colaboradores, nacionales y extranjeros, como mentores, compañeros de mentores y profesionales críticos ingleses, lacreación de una red de apoyo para apoyar esta actividad. Palabras clave: Ciencia. Investigación. Publicación Científica. Idioma en Inglés. Análisis de Contenido.

\section{INTRODUÇÃO}

A publicação de resultados de pesquisas em periódicos científicos de alto impacto é reconhecida como uma forte medida de sucesso acadêmico na atualidade, e tem se tornado uma realidade cada vez mais presente. Vários países, entre eles o Brasil, tem buscado ampliar o número de publicações em língua inglesa, de maneira a alcançar uma internacionalização através do aumento de citações em periódicos de visibilidade internacional.

Existem diferentes bases de dados científicos nas quais tais periódicos podem ser encontrados e que avaliam de formas diferentes os artigos neles publicados, como por exemplo: o PubMed/ 
Medline que apresenta artigos na área das ciências biomédicas, permite o maior número de palavras por busca e é atualizado diariamente; o Scopus, de propriedade da Elsevier, que se proclama como o maior banco de dados de resumos e citações de literatura de pesquisa e seleçãode resultados, cobrindo 27 milhões de resumos, 230 milhões de referências e 200 milhões de páginas na web; o Google Acadêmico, ferramenta que permite uma busca da literatura acadêmica, incluindo teses, livros, resumos e artigos feitos por toda a web com ajuda do buscador Google do qual se origina; e o JournalofCitationReports(JCR/ISI Web ofKnowledge) da Thompson Reuters Scientific, que inclui artigos desde 1900 até os dias atuais, entre outras bases.O JCR/ISI Web ofKnowledgedestaca-se por ser uma base de dados científicos que segundo Falagaset al. ${ }^{1}$ foi concebida com a intenção de satisfazer seus usuários na análise de citações. Essa base de dados permite análises bibliométricas, oferecendo ferramentas para análises de citações, referências e índice h que quantifica o número de vezes em que os artigos do pesquisador são citados. Ela tem dominado a área acadêmica, principalmente por lançar anualmente uma listagem de índices de fator de impacto dos periódicos em todas as áreas do conhecimento. Estes índices vêm sendo utilizados para classificar o grau de influência e importância dos grupos de pesquisa e pesquisadores e também por instituições que precisam avaliar as formas de ingresso e progresso na carreira acadêmica e a produtividade em pesquisa, como os programas de pós-graduação stricto sensoda área de ciências da saúde. No âmbito desses programas de pós-graduação já tem sido adotado como indicador para medir a produtividade de um pesquisador o índice h.

Neste contexto de possibilidades de internacionalização imediata dos achados científicos e de alta produtividade, umas das subáreas das ciências da saúde ligada ao conhecimento biomédico que mais tem se destacado no cenário brasileiro é a das neurociências e psiquiatria. Nos últimos anos, ainda que se mantenha relativamente estável o número anual de publicações por autores brasileiros nas neurociências e psiquiatria, tem ocorrido um aumento expressivo da proporção de artigos com maior visibilidade internacional, publicados em periódicos de maior impacto, delíngua inglesa ${ }^{2}$. Atualmente, os principais periódicos biomédicos brasileiros vinculados a programas de pósgraduação na área de ciências da saúde,como por exemplo, Clinics, Revista Brasileira de Psiquiatria e BrazilianJournalof Medical andBiologicalResearch, aceitam artigos somente em inglês.

Além da redação do próprio conteúdo do artigo, o domínio da língua inglesa nos meios acadêmicos em países nos quais os pesquisadores não tem a língua inglesa como idioma oficial envolve dificuldades. Isso ocorre inclusive em países com destaque e tradição científica como a França e a Alemanha, ou tecnológica como Coréia e Japão ${ }^{3}$.

Quais são as dificuldades e estratégias de pesquisadores brasileiros da área de ciências da saúde na preparação de um artigo em inglês e publicação dos resultados de suapesquisa em um periódico de língua inglesa? 
$\mathrm{Cunha}^{4}$, em recente pesquisa de caráter quantitativo realizada com estudantes de pós-graduação stricto sensu de psiquiatria da Faculdade de Medicina da Universidade de São Paulo (FMUSP), demonstrou através de análises de regressão múltipla, que eles tinham altos índices de conhecimento da língua inglesa, verificado tanto pelas notas obtidas em um teste objetivo de conhecimento utilizado para admissão do estudante na pós-graduação, como pela autoavaliaçãodestes estudantesque indicava bom nível de autopercepção a respeito da competência no uso da língua inglesae constatouque o conhecimento de inglês não é o fator chave quando o assunto é o sucesso das publicações (aceitas por periódicos considerados relevantesnas ciências biomédicas).Outras variáveis (o índice h dos orientadores e tradução deterceiros e revisão do inglês) também exercem influência como preditoressignificativos de tal sucesso ao publicar (Cunha et.al, no prelo).No presente estudo, descrevemos os resultados qualitativos obtidos na mesma amostra de estudantes, avaliando respostas dadas pelos mesmos ao buscarem soluções para publicar em inglês.

O programa de pós-graduação ao qual estes estudantes estão vinculados, apresenta regras rigorosas no que diz respeito ao incentivo à publicação dos resultados das pesquisas acadêmicas. Os estudantes (mestrado e doutorado) só podem defender seus trabalhos se tiverem submetido os resultadospara publicação em periódicos indexados. Caso necessitem, todos os pós-graduandos têm acesso a serviços gratuitos para revisão (e não tradução integral) de seus artigos científicos para língua inglesa. Nenhum estudante pode se matricular para o doutorado se não tiver artigo aceito para publicação e os orientadores são avaliados trienalmente em termos da quantidade e impacto de suas publicações com base no JCR/ISI Web ofKnowledge, podendo ser descredenciados se sua produção em língua inglesa, indexada nessa base de dados, for considerada insuficiente.

\section{MÉTODO}

Estudo de carácter qualitativo descritivo utilizando dados de duas questões abertas do questionário aplicado por $\mathrm{Cunha}^{4} \mathrm{em}$ uma amostra de alunos de pós-graduação de psiquiatria, que obtiveram seus títulos entre julho de 2009 e maio de 2011, onde descrevemos e analisamos as dificuldades e estratégias empregadas pelos estudantes do programa de pós-graduação em psiquiatria da FMUSP para escrever e publicar artigos, derivados de seus trabalhos de mestrado e doutorado, em periódicos indexados pelo JCR-ISI Web ofKnowledge. De um total de 46 possíveis sujeitos, 43 responderam ao questionário proposto pelo autor, sendo 16 do sexo masculino (37.2\%) e 27 do sexo feminino (62.8\%), dos quais 28 cursavam mestrado e 15 o doutorado, e que obtiveram a titulação entre julho de 2009 e maio de 2011.

Os estudantes acessaram o questionário via internet respondendo questões fechadas sobre o histórico prévio com a língua inglesa; a autoavaliação dos conhecimentos nessa língua; entre outras. Além disso, o questionário incluiu questões abertas visando aprofundar o posicionamento dos estudantes sobre como lidam com as dificuldades, tais como: "De forma geral e independentemente 
de ser em português ou inglês, você diria que escreve bem?"; "Qual foi o nível de dificuldade encontrado por você ao redigir o primeiro artigo científico em língua inglesa relacionado à sua tese (ou dissertação)?”.

Realizamos uma análise de conteúdo de caráter qualitativo das respostas dos estudantes às duas questões acima, de modo a considerar a presença ou ausência de uma dada característica de conteúdo ou conjunto de características num determinado fragmento da mensagem ${ }^{5}$. Assim, foi possível agrupar os principais significados emergentes em categorias separadas, construídas com base na convergência dos conteúdos que surgiram nas respostas.

\section{RESULTADOS}

O conteúdo das respostas chamou atenção pela variedade e riqueza de informações. Os significados que emergiram deram origem e foram agrupados nas categorias: autoavaliação da habilidade na redação/escrita; dificuldades encontradas na preparação do artigo em outra língua; e rede de apoio para confecção de artigos. A primeira categoria expressa conteúdos sobre como eles entendem seu conhecimento e manejoda língua. A segunda categoria refere-se aos obstáculos e impedimentos de toda ordem no processo de redação do artigo em língua estrangeira.A terceira categoria, por sua vez, refere-se aos recursos materiais, humanos e técnicos que subsidiam o processo de escrita.

\section{- Autoavaliaçãodos estudantes sobre sua habilidade na redação/escrita}

As respostas mostram uma autoavaliaçãopositiva em termos de domínio da língua portuguesa e inglesa, permitindo identificar como esse grupo se vê ao produzir um material escrito. A grande maioria afirmou escrever bem e utilizar a língua portuguesa e/ou inglesa de forma competente como ilustram as respostas dos estudantes $01,02,05$ e 09 :

(1)R: Sim, escrevo muito bem. Sempre gostei muito de português e sempre foi a disciplina em que eu me saía melhor na escola. O inglês foi uma consequência, pois sempre gostei de línguas e sempre tive facilidade em aprender, o que me motivou a estudar não só o inglês, mas também o espanhol.

(2)R: Sim. Acredito que meu vocabulário e minha articulação sintática permitam que eu me comunique de forma eficiente por meio da linguagem escrita.

(5)R: Escrevo bem, pois tenho forte preocupação em me inteirar conceitualmente sobre o tema que vou discorrer. Além disso, sou atenta a questões gramaticais e estruturais. 
(9)R: Sim, pois procuro escrever de forma correta, tanto semântica quanto gramaticalmente, trabalho as frases buscando tornar o texto o mais claro possível, reproduzindo de maneira fidedigna o meu pensamento.

Tem habilidade na escrita aqueles que conhecem o português, se comunicam (falam) melhor, gostam de ler e de escrever. Aqueles que estão bem em português tendem a se sair bem no aprendizado de outras línguas também. As respostas dos estudantes 21, 28, 30 e 32, mostram o quanto eles avaliam positivamente seus conhecimentos de escrita:

(21)R: Sim. Sempre tive muita facilidade de escrever e sempre fui muito elogiada a respeito da qualidade dos meus textos.

(28)R: Sim. Acredito que a escrita é uma de minhas principais habilidades. Na minha defesa de mestrado, fui elogiada pela banca pela qualidade e coerência do texto da dissertação.

(30)R: Sim. Minha escrita tanto em inglês quanto em português é clara, coerente e concatenada. Tenho o costume rotineiro de escrever textos (não obrigatoriamente científicos) e poesias, algo que é muito valioso para ajudar a manter a arte de se expressar bem através da escrita. A leitura, obviamente, também faz parte das minhas atividades favoritas e, sem dúvida, colabora para a melhora do vocabulário, escrita e expressão de meu pensamento.

(32)R: Tenho habilidade e conhecimento acima da média em gramática portuguesa e mediana em língua inglesa. Considero que tenho facilidade e interesse por línguas, no entanto, conheço bem algumas estruturas mais formais e literárias em língua inglesa. Sinto dificuldade para falar mais informalmente o inglês. Na área científica, a leitura dos textos é facilitadora, mas o formato é repetitivo. Outra questão é ter de escrever ora para revistas americanas, ora inglesas, o que exige mais conhecimento.

A resposta do estudante 21 revela como o reforço, na forma de elogio, é importante para autoconfiança na redação em português, aspecto que não apareceu ou não foi evidenciando nas respostas dos estudantes em geral ao comentar o processo de redação em língua inglesa. A resposta do estudante 32 mostra que ele conhece bem a língua inglesa, sobretudo, quando se considera o conhecimento da língua para situações de formalidade ou situações de vida cotidiana. Ele também aponta as diferenças de se escrever em inglês para periódicos americanos ou ingleses, o que certamente demanda maior conhecimento da língua e também de aspectos culturais implícitos.

Por outro lado, a tarefa de escrever em língua estrangeira também se coloca como um desafio gerador de ansiedade para os estudantes porque traz a tona o medo de não ser compreendido, como 
mostra a resposta da estudante 07:“Acho que escrevo bem, porque sempre gostei de ler, tanto em inglês como em português e sempre fui muito preocupada em ser bem compreendida[...]”.

\section{- Dificuldades encontradas pelos estudantes na preparação do artigo em língua inglesa}

Os estudantes focalizaram o conteúdo de suas respostas no problema que a pouca proximidade com a escrita científicapode gerar na hora de se preparar um artigo em inglês. $O$ estudante 10 destacou a contribuição da pós-graduação no aprimoramento da sua escrita científica:“Acredito que o mestrado e o doutorado propiciaram que eu escreva bem, com vocabulário adequado e seguindo as regras necessárias".

As respostas abaixo dos estudantes 14 e 37 mostram como se dá a relação com a escrita científica e a necessidade constante de trabalhá-la para o domínio da mesma:

(14)R: [...]os desafios da escrita científica tornam necessário um contínuo aprimoramento. A habilidade da escrita é exercitada de modo cotidiano e nos últimos tempos tenho me organizado para fazê-lo.

(37)R: [...]Considero que minha escrita é boa porque procuro expressar as informações de forma clara, seguindo uma linha de raciocínio. Além disto, acredito que tenho bom conhecimento de regras gramaticais.

Utilizar outra língua de forma científica e correta implica em enfrentar dificuldades que envolvem desde a escolha da língua de pertença do periódico (inglês americano, bretão, australiano, sul africano), até a precisão na tradução de termos e conceitos. Os trechos apresentados a seguir retirados das respostas dos estudantes $01,03,07,16,23,32$ e 39, indicam que o uso da linguagem técnica e, consequentemente, a escrita científica são uma das principais dificuldades encontradas pelos estudantes:

(1)R: Tive dificuldades com alguns termos técnicos.

(3)R: Grau de dificuldade moderado. Principalmente para termos técnicos que depois foram incorporados.

(7)R: A dificuldade maior foi encontrar os termos adequados para descrever a metodologia estatística.

(16)R: Tive dificuldade em adaptar os termos científicos e as expressões utilizadas[...].

(23)R: A linguagem científica colocada de forma resumida. 
(32)R: A dificuldade maior foi com a linguagem científica.

(39)R: Dificuldades relacionadas a termos técnicos/científicos.

\section{- Rede de apoio para confecção de artigos}

Os estudantes possuem uma visão de que o auxílio de terceiros é fundamental para sucesso na confecção do artigo. O conteúdo das respostas dos estudantes 02, 24, 25, 31 e 43 a questão "Qual foi o nível de dificuldade encontrado por você ao redigir o primeiro artigo científico em língua inglesa, relacionado à sua tese (ou dissertação)?”, revela que a segurança para a escrita em língua inglesa só existe quando há participação de outras pessoas:

(2)R: [nível de dificuldade] Alto, precisei de um revisor para adequar o texto.

(24)R: Eu tive dificuldade mediana, sendo necessária revisão por outro profissional para que me sentisse segura.

(25)R: Nível médio [de dificuldade]; sabia a mensagem que gostaria de passar, mas só me sinto segura com a revisão de um profissional.

(31)R: [nível de dificuldade] Alto. Uma tradutora traduziu o texto do português para o inglês.

(43)R: Foi importante, necessitei de revisores nacionais e internacionais para ficar completamente compatível com a língua inglesa.

A presença do orientador é entendida como um fator que possibilita maior confiança, desempenhando um papel fundamental no momento de preparação da redaçãodo artigo, como mostra as respostas dos estudantes $05,10,12$ e 33 :

(5)R: [...] Minha orientadora sempre orientou que artigos científicos devem ser escritos diretamente na língua inglesa, o que torna o artigo mais fluido, porém precisa de mais tempo para ser elaborado.

(10)R: Tive a felicidade de poder contar com grande ajuda do Dr. X [orientador] ao redigir meu primeiro artigo, caso contrário, só teria conseguido se tivesse contratado ajuda profissional.

(12)R: [...] Tive uma coorientadora dos EUA, portanto as dificuldades com gramática e termos eram prontamente resolvidas durante as discussões do artigo - via 
e-mail, Skype ou pessoalmente.

(33)R: [...] pedi ajuda ao meu professor, além da minha orientadora, para a revisão do artigo.

Essa busca de auxílio de terceiros, ao menos parcial, para a confecção de artigos em inglês indica que o sucesso nas publicações em periódicos de maior impactodemanda conhecimentos de escrita técnica em língua estrangeira que não são cobertos pelo tipo de conhecimento prévio mais genérico da língua que os estudantes trazem em sua bagagem. O que aumenta as dificuldades frente àtarefa de receber o parecer do artigo em outra língua, e a necessidade de responder ao mesmo articulando simultaneamente aspectos técnicos e de conteúdo.

\section{DISCUSSÃO}

No Brasil os pesquisadores tem alcançado nos últimos anos um nível melhor de utilização da língua inglesa em publicações científicas, comprovado pelo crescimento do número de publicações em periódicos de alto impacto. Isso mostra que houve um aumento da qualidade em pesquisa e que ela tem sido avaliada não só como conteúdo científico, mas também em termos de utilização adequada da língua inglesa enquanto língua franca de difusão do conhecimento científico e, consequentemente, atenção e reconhecimento internacional.

Estudos como os de Meneghini\& Packer ${ }^{6}$, Vasconcelos $^{7}$ e Volpato ${ }^{8}$ tem investigado as dificuldades de publicar em língua inglesa no Brasil e como o sucesso dessas publicações é configurado. Estes autores destacam fatores como dificuldades de escrita científica e de adequação à língua (inglês americano versus inglês britânico), além de outros fatores que podem ser associados com a formação científica no Brasil e o problema de ter que produzir em língua inglesa sem um suporte adequado (infraestrutura, cursos de redação do texto acadêmico, apoio financeiro para revisões, entre outros).

Nas respostas dos estudantes de pós-graduação da área de ciências da saúde na especialidade de psiquiatria, foi possível identificar como principais dificuldades para a produção de artigos científicos em língua inglesa: a falta de treino na escrita em inglês; a compreensão e cumprimento de todos os padrões e normas estabelecidos pelos periódicos; a tradução de termos científicos. Suas principais estratégias para produzir os artigosenvolvea leitura de outros artigos em língua inglesa; a redação do artigo desde o início em inglês; e a organização de uma estrutura básica do texto (introdução, resultados, conclusão). Além disso, para cumprimento dessa tarefa os estudantes têm contado com a presença de revisores, nacionais e estrangeiros, como orientadores, co-orientadores e revisores profissionais em língua inglesa.

Embora as respostas tenham referido bom conhecimento da língua inglesa, verificado inclusive por prova objetiva para ingresso na pós-graduação, e avaliação positiva da capacidade de escrita no idioma, o contexto de produção das respostas mostra que não é tarefa fácil transformar o 
conhecimento do idioma em um artigo científico (linguagem científica, formato dos periódicos, inglês britânico versus americano)apto a ser aprovado para publicação em periódicos de relevância internacional.

A autoconfiança no domínio de um idioma não se traduziuem autonomia para produção científica nesse idioma, sendo necessária a participação de terceiros(orientadores, coorientadores, revisores, entre outros), que configuram uma rede de apoio e suporte a essa atividade.

O conhecimento da língua inglesa é importante para redação dos artigos, mas também é preciso levar em conta a necessidade de maior preparo dos pesquisadores na redação do texto acadêmico de modo geral, o que deveria incluir como diz Volpato ${ }^{8}$ desde uma formação em filosofia até o conhecimento de conceitos científicos.

Nesse sentido, é importante que ao ingressar na pós-graduação os estudantes de ciências da saúde possam ser preparados/orientados/treinados desde o início para a tarefa da redação científica, com disciplinas específicas que possam auxiliar na redação em inglês com termos técnicos da área e de metodologia. Conceitostanto do que seja ciência quanto dosmétodos para a produção científica também podem sermais amplamente explorados. Assim como a exploração da escrita científica em português já levando em conta que o mesmo manuscrito deve ser produzido em língua estrangeira para submissão e consequente publicação em periódicos de relevância científica internacional.É fundamental formalizar a importância da participação dos estudantes de pós-graduação em cursos de redação de artigos em português e inglês ${ }^{8,6}$, ampliando sua oferta, incentivo e divulgação nosprogramas nacionais de pós-graduação stricto sensu da área de ciências da saúde.Buscamos identificar as dificuldades e estratégias dos estudantes da área na redação e submissão de artigo eminglês, com intuito de contribuir para essa discussão no âmbito dos programas de ciências da saúde e junto às políticas de formação de pesquisadores.

\section{REFERENCIAS BIBLIOGRÁFICAS}

1 Falagas ME, Pitsouni EI,Malietzis GA, Pappas G. Comparison of PubMed, Scopus, Web of Science, and Google Scholar: strengths and weaknesses. Journal FASEB.2008; 22: 338-342.

2Bressan RA, Gerolin Je Mari JJ. The modest but growing Brazilian presence in psychiatric, psychobiological and mental health research: assessment of the 1998-2002 period. BrazilianJournalMedical andBiologicalResearch. 2005; 38(5): 649-659.

${ }^{3}$ Gonzáles-Alcaide G, Valderrama-Zurian JC, Aleixandre-Benavent. The impact factor in noEnglish-speaking countries.Scientometrics2012; 92(2): 297-311.

${ }^{4}$ Cunha A. Relação entre conhecimento em língua inglesa e sucesso das publicações dos pós- 
graduandos de psiquiatria e neurociências em periódicos internacionais de impacto [Dissertação de Mestrado], São Paulo (SP): Faculdade de Medicina da Universidade de São Paulo; 2013.

${ }^{5}$ Puglisi ML, Franco B. Análise de Conteúdo. 2a Edição. Brasília: Liber Livro; 2005.

${ }^{6}$ Meneghini R, Packer AL. Is there science beyond English? Initiatives to increase the quality and visibility of non-English publications might help to break down language barriers in scientific communication. EMBO Rep. 2007; 8(2): 112-116.

${ }^{7}$ Vasconcelos SMR, Sorenson MM, Leta J. Scientist-friendly policies for non-native Englishspeaking authors: timely and welcome. Bras J Med Biol Res. 2007; 40(6): 743-747.

${ }^{8}$ Volpato GL. Ciência: da filosofia à publicação. $6^{\mathrm{a}}$ Edição. São Paulo: Cultura Acadêmica; 2013.

Artigo apresentado em 14/03/14 Artigo aprovado em 24/07/14 Artigo publicado no sistema em 12/09/14 